Cahiers d'études africaines

\title{
SHUBIN, Vladimir. - The Hot « Cold War » : The USSR in Southern Africa
}

\section{Luc Ngwe}

\section{OpenEdition}

\section{Journals}

Édition électronique

URL : http://journals.openedition.org/etudesafricaines/20787

DOI : 10.4000/etudesafricaines.20787

ISSN : 1777-5353

\section{Éditeur}

Éditions de l'EHESS

\section{Édition imprimée}

Date de publication : 1 juillet 2017

Pagination : 473-477

ISBN : 978-2-7132-2688-5

ISSN : 0008-0055

\section{Référence électronique}

Luc Ngwe, "Shubin, Vladimir. - The Hot "Cold War»: The USSR in Southern Africa », Cahiers d'études africaines [En ligne], 226 | 2017, mis en ligne le 01 avril 2017, consulté le 24 septembre 2020. URL http://journals.openedition.org/etudesafricaines/20787 ; DOl : https://doi.org/10.4000/ etudesafricaines.20787

Ce document a été généré automatiquement le 24 septembre 2020.

(c) Cahiers d'Études africaines 


\title{
SHUBIN, Vladimir. - The Hot « Cold War »: The USSR in Southern Africa
}

\author{
Luc Ngwe
}

\section{RÉFÉRENCE}

SHUBIN, Vladimir. - The Hot "Cold War» : The USSR in Southern Africa. London-Scottsville, Pluto Press-University of KwaZulu-Natal Press, 2008, 320 p., index, ill.

1 Les décolonisations, les luttes de libération nationale et leurs principaux acteurs en Angola, au Zimbabwe, en Mozambique, et en Namibie ainsi que la fin de l'apartheid en Afrique du Sud entre 1960 et 1990 durant «la Guerre froide» sont au centre des analyses de Vladimir Shubin. Celui-ci éclaire la présence de l'Union soviétique dans ces pays, et s'intéresse particulièrement aux formes qu'a prises son intervention dans les différentes luttes de libération nationale ou encore dans la lutte pour l'abolition de l'apartheid en Afrique du Sud et qui, selon lui, ont été insuffisamment étudiées et souvent déformées.

Divisé en cinq parties inégales qui traitent dans l'ordre d'importance et successivement des luttes de libération en Angola, au Mozambique, au Zimbabwe, en Namibie et en Afrique du Sud, The Hot "Cold War" est à mi-chemin entre l'étude scientifique que vérifie la dimension ethnographique des dynamiques de terrain et les mémoires d'un acteur. L'auteur n'affiche pas une grande ambition théorique, et ne prétend pas proposer une étude exhaustive des relations internationales de l'époque, de la Guerre froide, ou de l'ensemble des événements politiques survenus en Afrique australe. Il s'attache plutôt à restituer, dans leur complexité, les luttes de libération nationale ainsi que l'abolition de l'apartheid. Il adopte une démarche narrative qui s'appuie sur sa position d'acteur et de témoin des situations décrites. Directeur adjoint de l'Institut d'études africaines de l'Académie des sciences de Russie, Shubin a été successivement officier des forces armées soviétiques de 1962 à 1969, secrétaire du Comité soviétique de solidarité afro-asiatique, chef de sa section africaine de 1969 à 1979, et chef adjoint 
du Département des affaires étrangères du Comité central du Parti communiste de l'Union soviétique de 1982 à 1991. Troquant souvent son costume d'acteur pour celui de témoin, il a participé à différentes délégations officielles en Afrique australe, par exemple lors du premier anniversaire de l'indépendance de la Namibie en 1991 et, au cours de la même année, à la Conférence nationale de l'ANC (Durban, Afrique du Sud). La diversité de matériaux qu'il mobilise provient de ses notes personnelles, de ses souvenirs mais aussi des mémoires des acteurs de l'époque, auprès desquels il a recueilli de précieux témoignages, de la presse (Pravda), des archives russes officielles (Archives d'État de la Fédération de Russie [GARF], Archives russes d'État d'Histoire contemporaine [RGANI] ${ }^{29}$ et informelles (archives familiales), et sud-africaines (ANC et Parti communiste $)^{30}$.

3 Vladimir Shubin pose d'emblée le décor en soulignant que le soutien de l'Union soviétique aux luttes de libération nationale dès les années 1950 relevait de la lutte anticoloniale et anti-impérialiste contrairement, selon lui, à la croyance répandue chez les hommes politiques occidentaux ${ }^{31}$ et aux analyses des universitaires qui observaient les conflits armés en Afrique australe à travers le prisme déformant de la rivalité entre superpuissances. Ces conflits n'ont pas été de simples " guerres par procuration », mais bien une lutte contre l'impérialisme de l'ensemble du monde progressiste à laquelle participaient aussi bien la communauté socialiste, les mouvements de libération nationale que la classe ouvrière des pays capitalistes. D'ailleurs, soutient l'auteur, le terme «Guerre froide » ne faisait pas partie du vocabulaire politique soviétique. De la même façon, «la mentalité russe » avait, selon lui, une «sympathie naturelle » pour le plus faible et surtout pour une cause juste.

Bien plus, Moscou ne soutenait pas les mouvements de libération nationale en fonction de leur orientation idéologique communiste, mais en fonction de leur objectif de libération, de leur viabilité, de leur représentativité et de leur légitimité auprès des populations. D'ailleurs, les orientations socialistes suivies par plusieurs de ces mouvements et de leurs leaders étaient le fait de leur libre choix, sans exigence de loyauté, même si celle-ci était bien accueillie à Moscou. Ainsi, l'URSS a appuyé le Mouvement populaire de libération de l'Angola (MPLA), considéré comme seul représentant du peuple angolais plutôt que l'Union nationale pour l'indépendance totale de l'Angola (UNITA) de Jonas Savimbi et le Front national de libération de l'Angola (FNLA) de Roberto Holden. Au Mozambique, Moscou a accordé son aide au Front de libération du Mozambique (FRELIMO), même si la relation de celui-ci avec la Chine affectait sa proximité avec l'Union soviétique. Au Zimbabwe, c'est à l'Union du peuple africain du Zimbabwe (ZAPU) que l'Union soviétique a accordé ses faveurs dès les années 1960 et ceci au détriment de l'Union nationale africaine du Zimbabwe (ZANU) après sa scission avec le ZAPU en 1963 et son rapprochement avec la Chine.

En Namibie, l'URSS a d'abord été attirée par l'Union nationale du Sud-Ouest africain (SWANU), où l'intérêt pour les questions nationales primait sur l'intérêt pour les questions ethniques de l'Organisation du peuple du Sud-Ouest africain (SWAPO) qui mobilisait exclusivement dans le groupe ethnique Ovambo. Cependant, le soutien de Moscou s'est inversé lorsque la SWANU a adopté l'option chinoise à la fin de 1963 et est devenue moins légitime, tandis que la SWAPO, dont les dirigeants fréquentaient Pékin sans pour autant en suivre les orientations, est devenue plus nationale et ancrée dans la lutte de libération nationale. En Afrique du Sud, l'URSS n'a jamais ménagé son soutien 
au Congrès national africain (ANC) et au Parti communiste sud-africain (SACP) depuis leur fondation.

6 Par ailleurs, Shubin fait apparaître les luttes de libération dans toute leur complexité, bien à l'opposé de la perception fortement répandue selon laquelle les interventions soviétiques et plus globalement des pays du Pacte de Varsovie dans ces conflits obéissaient à la logique totalitaire qui présidait au fonctionnement interne du bloc de l'Est ou encore étaient sous-tendues par l'idéologie communiste. Selon cette perception, Cuba ou les pays africains, qui se rangeaient du côté des forces progressistes d'Afrique n'étaient que de simples satellites de Moscou. Or, les luttes de libération nationale s'inscrivaient, selon l'auteur, dans des configurations et des dynamiques propres à chaque pays. Ainsi, les conflits armés en Afrique australe comportent une diversité d'acteurs mus par des enjeux et des intérêts différenciés. À l'aide des documents d'archives et de ses expériences sur le terrain, il affirme que la décision cubaine d'intervenir en Angola était bien éloignée des injonctions soviétiques, d'autant que les deux pays, Cuba et l'URSS, divergeaient souvent sur les stratégies militaires adoptées. De la même façon, les pays africains ont joué différents rôles dans ces processus politiques bien loin de l'union de façade et des déclarations de principe sur la décolonisation proclamées par l'Organisation de l'unité africaine (OUA) et par les différents pays du continent. Ainsi en Angola, les pays limitrophes et parfois alliés des différents blocs constitués, à l'instar du Congo, du Zaïre ou de la Zambie, jouaient leur propre partition dans la confrontation sans répondre d'un quelconque alignement idéologique. Que dire alors de l'alliance entre les États-Unis et la Chine qui soutenaient l'UNITA contre le MPLA, allié de l'Union soviétique au moment de l'indépendance en Angola? Cette coalition sino-américaine s'est heurtée à la résistance du Mozambique et de la Tanzanie.

7 Last but not the least, Shubin démonte avec vigueur une autre fausse idée occidentale selon laquelle l'Union soviétique constituait un bloc monolithique dont le fonctionnement strictement vertical n'obéissait qu'à des considérations idéologiques. Il mentionne alors l'absence d'homogénéité au sein de l'appareil de décision de Moscou, notamment entre les instances politiques et le KGB; prenant à titre d'exemple les dissensions entre les différentes autorités soviétiques, à la suite de l'initiative de Petr Yevsyukov, alors ambassadeur au Mozambique, de rencontrer Mugabe. Ainsi le problème angolais et, par extension, les conflits armés en Afrique australe n'ont pas été traités par des instances institutionnelles impersonnelles agissant uniquement en fonction de la logique idéologique, mais par des considérations pragmatiques, soumises parfois aux «zigzags» de l'histoire ainsi qu'aux rapports personnels d'amitié ou d'animosité avec les leaders de ces mouvements nationalistes. Ces derniers ne manquaient pas d'influer sur les décisions et les actions.

Shubin donne tout au long de l'ouvrage des informations assez précises sur l'aide qu'a apportée l'Union soviétique à ces mouvements selon la situation dans chaque pays. Si cette aide était multiforme, notamment financière, logistique, d'accompagnement politique et diplomatique, elle a été essentiellement militaire. Revêtant plusieurs formes, elle concernait notamment la formation militaire des combattants, la fourniture des armes, l'encadrement technique via les conseillers et les spécialistes militaires, ainsi que la logistique. C'est ce soutien militaire qui a été décisif dans les processus politiques, particulièrement au Mozambique et en Afrique du Sud. L'auteur 
estime même que la fourniture d'armes lourdes au FRELIMO au Mozambique a contribué à décourager l'Afrique du Sud dans sa menace nucléaire.

De la même façon, l'intensification de la libération en Afrique du Sud et la reconnaissance croissante de l'ANC avec l'appui de Moscou pendant les premières années de la perestroika de Gorbatchev en 1986 ont créé une atmosphère de négociations favorable à un règlement politique et à l'élimination de l'apartheid par les moyens pacifiques.

10 En définitive, Shubin souligne que la plupart des conflits dans le monde pendant la Guerre froide ont davantage obéi aux dynamiques internes des pays et des régions qu'aux considérations idéologiques inspirées par la rivalité exacerbée entre l'Est et l'Ouest. Les conflits armés en Afrique australe ont été engendrés par la volonté populaire d'en finir avec le colonialisme et l'apartheid. Tout en concédant que l'implication des superpuissances a souvent donné une autre dimension aux conflits, Shubin soutient que l'intervention de l'URSS dans ces luttes, et particulièrement dans la formation des combattants, a été « salutaire » et qu'elle a pu dissuader les dirigeants des mouvements nationalistes d'utiliser les « méthodes terroristes ».

11 L'ouvrage de Shubin apporte des éclairages importants sur les processus politiques en Afrique australe. Il nuance le regard porté sur ces événements en termes de blocs en tempérant la lecture en vigueur d'une opposition tranchée Ouest/Est ou capitalisme/ communisme, et montre les dynamiques pratiques qui s'y déploient à travers l'alliance dans certaines situations entre les États-Unis et la Chine contre l'URSS ou encore l'affrontement sino-soviétique. The Hot "Cold War" éclaire aussi le caractère multilatéral des relations internationales des pays africains dans ce contexte ainsi que les enjeux propres, notamment de politique intérieure, qui inspiraient leur investissement dans ces conflits. En l'occurrence, comment comprendre l'engagement de l'ex-Zaïre de Mobutu dans ces conflits sans prendre en considération la question du kibanguisme?

12 Le récit de l'intervention soviétique dans ces conflits n'en est pas moins partial. Mises à part quelques réserves sur les atermoiements de l'URSS dans le choix de ses soutiens en Angola (Agostinho Neto ou Daniel Chipenda), ses prises de décision ou encore ses responsabilités dans les difficultés de la coopération avec le Mozambique après son indépendance, Shubin ne fait aucune critique sérieuse de cette politique. La présentation de situations, souvent à sens unique, dessert son auteur, qui se trouve victime d'une illusion, loin de l'examen objectif d'une situation critique qui aurait éclairé le rôle de l'URSS dans ces conflits, d'autant plus que la conjoncture était parfois inextricable.

13 À la suite de la lecture de ces pages, on peine à suivre Shubin dans sa vision philanthropique de l'intervention soviétique, et plus globalement de la présence de l'URSS dans ces pays et/ou dans ces conflits dans un contexte où la lutte acharnée entre deux camps pour la domination du monde autorisait tous les coups. La multiplication des alliés constituant une ressource importante dans cette domination, les mouvements de libération nationale formaient une des avancées du front de lutte, qualifiée d'antiimpérialiste par les pays du bloc de l'Est. Que dire des intérêts multiples qui se déployaient, ou qui sous-tendaient ces interventions et qui s'exprimaient dans les différents accords de coopération militaire, économique, culturelle? 
14 Par-delà les réserves énoncées ici, l'ouvrage de Shubin constitue un document riche en matériaux de travail, particulièrement pour les historiens.

\section{NOTES}

29. Shubin note qu'il n'a pas pu avoir accès à plusieurs des documents les plus pertinents dans les archives car ils n'ont pas été déclassifiés.

30. L'ouvrage comporte de nombreuses notes ainsi qu'un index des noms propres mais pas de bibliographie.

31. L'auteur cite l'exemple d'un document du Foreign Office britannique relatif à une rencontre en 1961 entre l'administration américaine sous Kennedy et les officielsbritanniques. 